\title{
EL APRENDIZAJE-SERVICIO EN LA FAMILIA PROFESIONAL DE SERVICIOS SOCIOCULTURALES Y A LA COMUNIDAD DESDE LA PERSPECTIVA DE LOS DOCENTES
}

\author{
SERVICE-LEARNING IN THE PROFESSIONAL FAMILY OF SOCIO- \\ CULTURAL AND COMMUNITY SERVICES FROM THE PERSPECTIVE OF \\ TEACHERS
}

\author{
Fátima Anaya Ruiz, Belén Suárez Lantarón, Ana López Medialdea \\ Universidad de Extremadura
}

\begin{abstract}
Correspondencia: Belén Suárez Lantarón
Correo: bslantaron@unex.es

Recibido: 28/01/20; Aceptado: 09/06/2020

DOI: $10.17398 / 0213-9529.40 .1 .35$
\end{abstract}

\begin{abstract}
RESUMEN
Este trabajo presenta el resultado de la investigación realizada como Trabajo Fin de Máster, cuyo objetivo ha sido describir la experiencia vivida por un grupo de docentes que han aplicado el aprendizaje-servicio (ApS) como metodología de enseñanza en la familia profesional de Servicios Socioculturales y a la Comunidad. Para alcanzar dicho propósito se utilizó una metodología cualitativa, siendo la entrevista el instrumento elegido en la obtención de la información. Los resultados obtenidos indican que la mayoría ha aplicado la metodología, aunque no reconocía su nombre, consideran que permite una aproximación del aprendizaje con realidades profesionales y que, aunque su puesta en marcha no es sencilla, el alumnado se implica y mejora los resultados de aprendizaje. Estas razones nos llevan a concluir que la aplicación del ApS en las aulas conlleva satisfacción por parte del profesorado y motiva y mejora los resultados de aprendizaje del alumnado.

Palabras clave: educación; formación profesional; aprendizaje-servicio; metodologías activas
\end{abstract}

\begin{abstract}
This paper presents the result of the research carried out as Master's Final Project, whose objective is to describe the experience lived by a group of teachers who have used service-learning (ApS) as a teaching methodology within the professional family of Services Sociocultural and in the Community. The methodology used to achieve this purpose was a qualitative methodology and the interview was the instrument chosen to obtaining the information. The analysis of the information was structured in relation to three main categories. The results obtained indicate that the majority have applied the methodology, although it did not recognize its name, they consider that it allows an approximation of learning with professional realities and that, although its implementation is not simple, the students are involved and improve the learning results. These reasons lead us to conclude that the application of ApS in classrooms entails satisfaction on the part of teachers and motivates and improves student learning outcomes.

Keyword: Education; vocational training; service-learning; active methodology.
\end{abstract}




\section{INTRODUCCIÓN}

En estas últimas décadas la educación ha sufrido transformaciones en un intento por adaptar la adquisición de habilidades y de conocimiento a los requerimientos de una sociedad del siglo XXI, buscando lograr el fin último de la educación que, como recoge nuestra legislación, se traduce en dos cuestiones (Uruñuela, 2018):

- La primera, lograr el desarrollo formativo del alumnado, su expediente académico adquiriendo aprendizajes sólidos que le hagan alcanzar un buen futuro profesional cuando llegue a la etapa adulta.

- La segunda, persigue el desarrollo integral de los estudiantes, es decir, de todas las dimensiones de la persona, sin limitarse a los aspectos académicos. Formar personas respetuosas, tolerantes, comprometidas, etc.

Ambas cuestiones, como explica Uruñuela (2018), no son excluyentes, planteando a los docentes el reto de cómo integrar ambas cuestiones en el desarrollo de su ejercicio profesional.

Por otro lado, en el ámbito educativo son muchas las situaciones y problemas que preocupan: el fracaso escolar, absentismo o abandono escolar; es fácil observar cómo existe entre el alumnado poco interés hacia contenidos y materias que no encuentran significación y utilidad para su día a día (Batller, 2013); los problemas de convivencia y respeto, la falta de valores (en algunos casos), las conductas disruptivas, el clima que se vive en las aulas (especialmente en las últimas etapas de la educación obligatoria y Formación Profesional, como explica Uruñuela, 2018).

Estas situaciones plantean la necesidad de adoptar cambios en las prácticas educativas que permitan dar una respuesta más adecuada para el logro de los fines antes descritos. Este proceso pasa por introducir nuevas fórmulas y metodologías de enseñanza-aprendizaje en las que se establezca una conexión entre teoría y práctica y el estudiante adopte un papel más activo (Torres y Vallejo, 2018).

En la misma línea, Suárez y Castillo (2020) indican que en este contexto surgen nuevas metodologías en las que cobra mayor protagonismo el estudiante y que buscan una formación integral y socialmente responsable, además, proponen resolver situaciones profesionales reales o ajustadas a situaciones que pueden darse en la vida real. Algunos ejemplos de dichas metodologías son: el aprendizaje basado en proyectos, los proyectos sociales, el aprendizaje por retos o el aprendizaje-servicio.

\section{Aprendizaje-servicio}

En los últimos años existe un fuerte movimiento pedagógico orientado hacia la educación para la ciudadanía al que se vincula el aprendizaje-servicio (ApS).

Este se define como un método de enseñanza-aprendizaje mediante un servicio a la comunidad. Batller (2013) lo describe como "un método para unir éxito educativo y compromiso social: aprender a ser competentes siendo útiles para los demás" (p. 20). Y el Centre Promotor d'Aprenentatge Sevei de Cataluña (citados en Mendia, 2016, p. 4) como "una propuesta educativa que combina procesos de aprendizaje y de servicio a la comunidad en un solo proyecto bien articulado en el que los participantes se forman al trabajar sobre necesidades reales del entorno con el objetivo de mejorarlo". Es decir: "una combinación dual de aprendizaje académico y de servicio a la comunidad, que se refuerzan entre sí y son mutuamente beneficiosos" (Deeley, 2016, p.25).

Coincidimos con autores como Batller (2013), Deeley (2016) o Uruñuela (2018) en que se trata de un movimiento pedagógico que vuelve a las bases de la educación, cuya finalidad es encontrar sentido al proceso de enseñanza-aprendizaje formando a ciudadanos competentes y 
críticos con la realidad social y, todo esto, a través del aprendizaje de los contenidos que están marcados a nivel curricular).

En la misma línea, Mendia (2016) explica que el ApS se sitúa en la corriente innovadora de la educación, puesto que no se trata solo de una actividad "extra", no es una acción "improvisada", es una acción educativa intencional y planificada. El autor la describe, además, como evaluable, inclusiva, que pone en contribución todos los recursos personales, materiales y tecnológicos necesarios para el desempeño de la acción a favor de la comunidad en todos sus procesos (planificación, ejecución, comunicación, investigación o difusión).

Esta forma de trabajo está cogiendo impulso en los centros educativos y también dentro de las Universidades, para conseguir la intencionalidad nombrada anteriormente haciendo que el alumnado se sienta parte de la sociedad y pilar fundamental del funcionamiento de la misma (Aramburuzabala, 2013).

Las tres grandes características del ApS, como señala Tapia (citada en Mendia, 2016, p. 4) son: el protagonismo activo de los participantes, el servicio solidario destinado a atender necesidades reales y sentidas de una comunidad y los aprendizajes intencionadamente planificados.

Evidentemente:

El ApS no es "el método", ni la única propuesta educativa innovadora. En su modestia y simplicidad realiza propuesta propuestas colaboradoras y cooperativas, constituyéndose en una palanca potente para la transformación de las prácticas educativas en la escuela y en la comunidad. Abre puertas, facilita la construcción de itinerarios, ofrece oportunidades, construye ciudadanía (Mendia, 2016, p. 14).

Entre los resultados obtenidos utilizando el aprendizaje-servicio, Deleey (2016, p. 29) expone los siguientes:

- Es una herramienta pedagógica

- Aporta un sentido mejorado de la ciudadanía

- Los alumnos pueden obtener mejores logros

- Aumento de las habilidades intelectuales

- Cambio en las actitudes y valores de los alumnos vinculadas a la diversidad, justicia, cambio social y desigualdad

- Desarrollo personal

- Desarrolla habilidades vinculadas a la empleabilidad

Sin embargo, esta autora también señala, entre las sobras del ApS, el hecho de que su efectividad puede resultar dudosa en tanto en cuanto puede suceder que no todos los alumnos se beneficien del ApS y que los resultados de este no pueden ser predichos puesto que existen multitud de programas de aprendizaje y variadas experiencias de voluntariado.

Estas razones nos han llevado a centrar nuestro estudio en esta metodología y, en concreto, en la etapa educativa de la Formación Profesional, deseando conocer cuál es la percepción de los docentes que han utilizado dicha metodología en sus aulas.

\section{La Formación Profesional: la familia de Servicios Socioculturales y al Comunidad}

Cuando se habla de Formación Profesional (FP), se está hablando de una especialización de los estudios con la intención de mejorar la empleabilidad de las personas y la productividad de las empresas (Soler, 2014).

Actualmente, a través de los Fondos Sociales Europeos, la Formación Profesional en nuestro país está viviendo un momento de auge con iniciativas como Europa 2020, (publicada por la Comisión Europea, 2010, ampliada la agenda a 2030), una estrategia de la Unión Europea 
(UE) para el crecimiento y la ocupación, cuya principal finalidad es la de garantizar la recuperación económica en la UE. Y dentro de las cuales se apuesta por los estudios postobligatorios que forman a profesionales a niveles técnicos.

La fuerza que está cogiendo la FP en España lleva a analizar cómo se está trabajando en ella, cuya lucha principal es eliminar el estigma y la desconfianza que provoca entre la población este tipo de estudios. Idea que se muestra en algunas de las noticias publicadas sobre FP como, por ejemplo: "la FP sigue siendo el «patito feo» de la educación" (La Vanguardia, 2019); "más de la mitad de los padres reconocen tener «mala imagen» de la FP (Cadena Ser, 2019) o "la FP sigue lastrada por su mala imagen entre los padres en favor de la universidad" (Piedra, 2019).

Sin embargo, estas noticias se contradicen con la realidad que se vive dentro de las aulas. El aumento en el gasto educativo hacia la FP y su apuesta por la modalidad de formación Dual1 donde los procesos de enseñanza-aprendizaje del alumnado se realizan en alternancia entre el centro educativo y empresas relacionadas en el sector. Esta información se contrasta con noticias como "Los alumnos de Formación Profesional crecen el doble que los de Bachillerato" (Blanco, 2019); o "La FP sigue disparada y crece un 50\%, alcanzando este año 11.716 matrículas" (González, 2019)

Las leyes educativas que regulan la FP en España son la LOE 2/2006 de 3 de mayo y, en algunos casos y adaptándose aún, algunos ciclos formativos se rigen por la LOGSE 1/1990 de 3 de octubre. Aunque actualmente el sistema educativo se encuentra sujeta a la LOMCE 8/2013 de 9 de diciembre, en el caso de la Formación Profesional no modifica nada de lo que queda recogido en la Ley Educativa anterior, aunque sí que recoge la nueva modalidad de FP Dual.

La Formación Profesional en nuestro país se organiza por familias profesionales (existen un total de 26) en función de la especialización de sus estudios, donde se incluyen los distintos títulos de $\mathrm{FP}^{1}$.

En el caso de este estudio nos enfocamos en la familia profesional de Servicios Socioculturales y a la Comunidad (en adelante SSCC), compuesta de siete ciclos formativos, distribuidos en Formación Profesional Básica, de Grado Medio y Grado Superior, como se muestra en la tabla 1.

Tabla 1. Distribución de ciclos formativos de la familia profesional SSCC

\begin{tabular}{ll}
\multicolumn{1}{c}{ Nivel de estudio } & \multicolumn{1}{c}{ Titulación } \\
\hline FP Básica & Actividades domésticas y limpieza de edificios \\
\hline FP Grado Medio & $\begin{array}{l}\text { Técnico/a en Atención a personas en situación de } \\
\text { dependencia }\end{array}$ \\
\hline & Técnico/a en Educación Infantil \\
& $\begin{array}{l}\text { Técnico/a en Integración Social } \\
\text { Técnico/a en Animación Sociocultural y turística } \\
\text { FP Grado Superior }\end{array}$ \\
& Técnico/a en Mediación Comunicativa \\
& Técnico/a en Promoción de Igualdad de género \\
\hline
\end{tabular}

Fuente: Elaboración propia

Según se recoge en el Real Decreto 1128/2003, los estudios de FP se registran en el Catálogo Nacional de Cualificación Profesional ${ }^{2}$, existiendo, además, legislación específica que regula esta familia profesional, la cual centra sus estudios en el desarrollo de las personas en todos los estadios de su vida, desde la infancia hasta la vejez, promocionando la autonomía de las personas y su desarrollo personal. Es una familia profesional donde el ámbito social y el desarrollo de la comunidad son su eje vertebral.

Precisamente, consideramos que, en este ámbito educativo, las metodologías activas tienen un campo de trabajo amplio y, en concreto el ApS, pues se presta al desarrollo de las competencias profesionales, personales y sociales que se exigen al alumnado destinatario. 
Considerando lo expuesto anteriormente, este trabajo pretende describir la experiencia vivida por un grupo de docentes que han trabajado dicha metodología dentro de la familia profesional de Servicios Socioculturales y a la Comunidad.

\section{METODOLOGÍA}

Para poder ahondar en las cuestiones que se plantean como objetivo de esta investigación, es necesario, en primer lugar, sistematizar el método que se va a llevar a cabo (Bisquerra, 2016). De acuerdo con los propósitos planteados se ha seleccionado para el logro los mismos, una metodología de corte cualitativo, ya que nos permite conocer, analizar y describir las vivencias de docentes que desempeñan su función en la familia de SSCC de la FP y han participado o utilizado ApS en sus aulas.

El instrumento utilizado para la recogida de información ha sido la entrevista, al considerar que da un aporte fenomenológico a la investigación. Heidegger (2008) define la fenomenología como "todo lo relativo al modo de la mostración y la explicación" (p. 59).

Es decir, para la consecución de este objetivo se pretende describir las experiencias vividas por una persona o un grupo de personas acerca de un concepto o fenómeno, es por ello por lo que se le da un importante valor a las entrevistas, las cuales se han realizado de forma presencial, pudiendo recoger la intencionalidad de sus palabras y la transmisión de sentimientos que se muestran durante el desarrollo de las mismas.

Estas entrevistas tienen un diseño semiestructurado en el que las cuestiones planteadas se enfocan en conocer: la concepción que tiene el profesorado sobre el ApS, la justificación de su uso en esta familia profesional y la funcionalidad del ApS dentro del aula.

\section{Población y muestra}

Para conocer de primera mano la experiencia de los docentes de la familia profesional de SSCC que han utilizado ApS en sus aulas, se selecciona una población de 12 profesionales, siguiendo un criterio de muestreo no probabilístico de conveniencia (son accesibles para el entrevistador) e intencional (mantienen las características relevantes para la investigación: docentes que trabajan en la FP y en la familia seleccionada, además de haber utilizado la metodología ApS en sus aulas). La muestra final de participación la compone un total de 7 docentes.

\section{Análisis}

Tras la transcripción de las entrevistas y su codificación, se lleva a cabo un análisis cualitativo de datos estableciendo tres categorías principales:

- El docente conoce el concepto de ApS

- Justificación del uso de ApS en esta familia profesional

- Funcionalidad de dicha metodología en el aula

Al ser una entrevista semiestructurada, estas categorías se complementan con otras ideas (subcategorías) aportadas por los docentes durante el desarrollo de dicha entrevista.

La codificación temática de los datos cualitativos obtenidos se realiza de modo manual, como explican Wood y Smith (2017), para facilitar su interpretación y dotarlos de sentido, codificando los datos en una matriz de temas (ver ejemplo en anexo I). 


\section{RESULTADOS}

A lo largo de este apartado se presentan los resultados obtenidos del análisis de las entrevistas. Se exponen en relación con las siguientes categorías antes mencionadas:

a) Conocimiento del docente sobre el ApS

En relación con la idea que los docentes tienen del ApS los resultados nos indican que el $100 \%$ personas entrevistadas reconocen haber hecho uso de esta metodología durante toda su labor docente, pero que no conocían que recibía un nombre en concreto, por ejemplo:

Profesor 1 “....aunque yo no sabía del concepto de aprendizaje servicio, pero sin embargo, oriento toda mi docencia hacia mi alumnado para mejorar en tiempo real la vida de una serie de personas."

Profesora 2 “...Ehh estaba haciéndolo, pero sin ponerle nombre, porque va como intrínseco. Yo estoy dando esta parte del temario y resulta que podemos aplicarlo en el entorno donde estamos."

Al hilo de esto, se reconoce realizar proyectos ApS aunque de manera puntual y esa es la diferencia entre lo que se realizaba antes y ahora, la continuidad en el tiempo:

Profesoras 6 y 7 "...la continuidad, el unirlo muy específico a contenidos de la materia, con contenidos y con resultados de aprendizaje y criterios de evaluación. Nos vale para esto, nos vale para evaluar o nos vale para favorecer el aprendizaje."

b) Justificación y aplicación de ApS en las aulas de FP-SSCC

Los resultados muestran que los profesores consideran la importancia de aproximar al estudiante con las distintas realidades con las que se va a encontrar en un futuro profesional y, en este sentido, esta metodología logra este objetivo. Sirva como ejemplo lo expresado por docentes del Ciclo de Atención a personas en situación de dependencia:

Profesoras 3 y 4 “...vemos que el alumnado se implica un 200\% cuando se plantea un proyecto de este tipo. Se entiende más el desarrollo a nivel personal, social, su motivación aumenta, aprenden a que los contenidos vistos en clase tienen una aplicabilidad el día de mañana..."

Profesoras 6 y 7 "es que es muy diferente lo que se ve en el aula que la realidad que se vive en la residencia, porque hoy estoy trabajando con esta persona de esta manera y mañana no puedo porque le ha ocurrido algo, esa es la realidad de las residencias..."

Otro resultado que nos ofrece la realización de las entrevistas que el 60\% de profesores señala que la puesta en marcha de estos proyectos no es sencilla, encontrándose el alumnado desorientado durante la realización de las prácticas y, hasta que no llevan tiempo trabajando no son conscientes del aprendizaje

Profesora 2: "ah vale, que lo que estoy aprendiendo sirve para algo."

Además, el $100 \%$ del profesorado entrevistado señala sobre la organización de los proyectos en los centros educativos lo importante que es que el claustro entienda el valor de este tipo de actividades, que comprendan el esfuerzo que supone poner en práctica estos proyectos. Es importante para ello disponer de un claustro y departamento de personas implicadas:

Profesor 1: "Empezó en la cosa sin mucho entusiasmo por parte del resto del departamento, tampoco el equipo directivo."

Profesora 2: "Es un poco un trabajo de ingeniería" 
Los docentes destacan, también, la importancia de tener desdobles en las aulas para trabajar con el alumnado estos proyectos, ya que esto ayuda a tener dos profesoras dentro del aula para el mismo grupo de alumnado y el trabajo se puede dividir:

Profesoras 3 y 4 :

"- ¿Tenéis desdobles?

Sí, claro, aprovechamos para ir en el momento que estamos dos profesoras para ir."

Otras preocupaciones manifestadas por los docentes entrevistados son:

- La necesidad de estabilidad del profesorado. El volumen de personal interino en esta especialidad es bastante numeroso, implicando esto que gran parte del profesorado cambia de centro cada curso escolar.

Profesor 1: "el proyecto lo lleva en sí el instituto, pero también las personas, porque si las personas consiguen tirar del grupo eso va a hacer que el proyecto de aprendizaje-servicio, a la hora de llevarlo a la práctica sea un éxito".

Profesora 5: "siempre te queda la cosa de podríamos haber llegado a más o podríamos haber hecho más..."

- Cierta inquietud sobre la compatibilidad de esta metodología con la nueva modalidad de enseñanza Dual, mostrándose más reacios a que se pueda llevar a cabo ambas formas de trabajo:

Profesoras 6 y 7: "es que... el aprendizaje-servicio creo que tiene otra intencionalidad, creo. Busca otro tipo de competencias que no son las que la dual busca o se fija."

\section{DISCUSIÓN Y CONCLUSIONES}

Los resultados de las entrevistas realizadas al profesorado que ha vivido la experiencia del ApS dentro del aula arrojan datos muy similares en cuanto a inquietudes y necesidades para que los proyectos se puedan llevar a cabo y conseguir la finalidad que persigue esta metodología. Exponemos a continuación las ideas principales recogidas en relación con la aplicación de esta metodología en el aula de FP:

a) Conocimiento del docente sobre el ApS

Tras los resultados obtenidos podemos afirmar que el ApS es una metodología que lleva aplicándose en esta familia profesional prácticamente durante todo su recorrido, ya que incluso su propio nombre (Servicios Socioculturales y a la Comunidad) subraya el hecho de trabajar hacia o para la comunidad.

Además, se destaca el hecho de que esta metodología ha sido puesta en práctica en las aulas muchas veces desconociendo su terminología. Aspecto que coincide con lo señalado en otros trabajos en los que se señala que prevalece una disparidad en su comprensión y conceptualización (Martínez, 2007), multiplicándose los términos con los que la denomina (hasta 147 términos ha contabilizado Kendall, citado en Martínez, 2007) y la variedad de formas en las se entiende o confunde con otros proyectos solidarios (Tapia, 2006)

b) La mejora de las habilidades del alumnado dentro del aula y de la funcionalidad de los aprendizajes

Los docentes señalan satisfacción en la mejora de las habilidades del alumnado y del rendimiento en los aprendizajes tras la aplicación de esta metodología en sus aulas. Esta 
cuestión corrobora las afirmaciones de otros estudios en los que se considera que el aprendizajeservicio no es un fin en sí mismo, sino una herramienta para hacer posible que los contenidos curriculares puedan ser aprendidos de modo significativo y contextualizado (Martínez, 2007) o que el ApS es una propuesta metodológica especialmente efectiva para poner en práctica contenidos teóricos a la vez que se promueve la solidaridad y la responsabilidad (Folgueiras y Martínez, 2009)

Además, como se afirma en el trabajo de (Mayor, López y Solís, 2019) "las prácticas de ApS se constituyen como actividades complejas que quiebran la forma de enseñanza de los dispositivos escolares tradicionales, basada en aprendizajes declarativos abstractos, descontextualizados y de escasa relevancia social" (p. 157). Y en cambio, en las acciones de ApS los aprendizajes, al estar imbricados con el servicio favorecen la comprensión de la realidad sobre la que se va a intervenir.

Del mismo modo, los docentes entrevistados muestran su satisfacción con esta metodología, en cuanto que conecta al alumnado con experiencias o realidades que encontrarán cuando accedan al mundo laboral. Es decir, que el ApS ayuda a que se desarrollan competencias relevantes para la inserción en el mundo del trabajo, además de otras transversales o genéricas (Folguerias y Martínez, 2009)

c) La preocupación sobre la inestabilidad del profesorado

El profesorado entrevistado también destaca que, para asegurar el éxito de esta práctica es necesario tener un control de las materias que se implican, es necesario poseer un recorrido previo para poder llegar a los objetivos planteados, sin embargo, la inestabilidad del profesorado no favorece este hecho, como ya apuntan Betrián y Jové (2013).

La realidad de muchos centros educativos es que el número de profesorado interino es bastante elevado, este fenómeno acaba afectando a la calidad de la educación. Tiene especial repercusión dentro de la Formación Profesional, donde en cada una de las especialidades del profesorado que imparte materias tiene una media de doce módulos profesionales diferentes en los que se imparte docencia con contenidos diferentes y que, esa falta de estabilidad le lleva a no dominar todos los contenidos para poder trabajar acorde a lo que se solicita. Por tanto, se sugiere que quizá, además de la necesidad de afianzar plantillas, también se deben desarrollar planes de formación que favorezcan el conocimiento de este tipo de acciones e innovaciones.

d) El número de alumnos/as por aula

Otra cuestión que se apunta en las entrevistas realizadas es la importancia de disminuir la ratio de alumnado por aula. El hecho de poder permitir en determinados ciclos la creación de desdobles, donde existen dos profesores/as por módulo, facilita el trabajo dentro del aula y poder desarrollar proyectos o prácticas que impliquen el uso de una comunidad para el aprendizaje.

Estos resultados coinciden con afirmaciones recogidas en trabajos anteriores como el informe elaborado por la OCDE (2014) en el que se explicaba que los docentes que trabajan en aulas con ratio elevada (más de 25 alumnos/as por aula) no llegan a dedicar más del $75 \%$ de sus clases al aprendizaje, pues pierden mucho de su tiempo en establecer el orden en el aula.

Tampoco debemos olvidar que, la ratio alumnos/aula es un indicador que incide en la calidad y equidad de la enseñanza (Pascual, 2006).

e) La constante necesidad de mejora.

Se destaca la importancia que tiene difundir este tipo de prácticas y la formación sobre la misma para poder unificar criterios que ayuden a preparar proyectos sólidos, que ayuden a 
mejorar todas las habilidades que se debe desarrollar en el alumnado y hacer de estos personas competentes y críticas.

Sin embargo y pese a ser la metodología principal de estos estudios sociales, son muchas las trabas que aparecen en los propios centros educativos, empezando por el claustro de profesores donde existen distintas formas de trabajo, generan muchos prejuicios, uno de los roces más comunes es el adaptar los horarios del profesorado de ciclos para contar con espacio suficiente entre horas y poder salir del centro a realizar este tipo de actividades.

En último lugar, consideramos importante destacar la irrupción de la nueva modalidad de enseñanza FP Dual. Se trata de una modalidad de aprendizaje en la que el alumnado alterna tanto la formación en empresas como la formación en el centro educativo. Destacamos esta modalidad puesto que, en esta situación, se reduce el tiempo para desarrollar las prácticas de ApS. El profesorado manifiesta la incompatibilidad que esta modalidad de enseñanza presenta para aplicar esta metodología, ya que el tiempo que se precisa para la preparación de estos proyectos se ve limitada con la alternancia del alumnado en las empresas, quedándose reducido el tiempo de permanencia dentro del centro educativo.

El desarrollo de este trabajo, también ha contado con sus limitaciones: primero, en cuanto a la metodología utilizada, pues el número de docentes participantes puede no ser significativo para hacer una generalización de los resultados. Asimismo, el uso de metodologías cualitativas que aportan resultados de carácter subjetivo, en tanto que se registra la sensación, opinión y actitud, pero pueden no ser objetivos.

Por estas razones, y dado que el ApS está en auge y se trabaja en todas las etapas y que, además favorece a ciudadanos socialmente involucrados, se propone como líneas de investigación futuras las siguientes:

- La posibilidad de replicar el estudio haciendo partícipes a más docentes y centros de FP, de modo que los resultados puedan hacerse más generalizables, así como utilizar otras herramientas metodológicas que complementen la información a obtener, como pueden ser los cuestionarios.

- Dado el empuje que tiene la FP actualmente en nuestro país, se considera interesante conocer más sobre el uso del ApS en estos estudios postobligatorios, en cualquiera de sus familias profesionales, con la intencionalidad de dar a conocer diferentes alternativas y las posibilidades que para las personas que realizan estos estudios.

- Por otro lado, se propone conocer la relación existente entre la difusión de estas prácticas y metodologías activas a través de las TIC y si esto ayuda al profesorado a implicarse más aún en su práctica educativa, de manera que esta línea de investigación siga avanzando con la finalidad siempre de mejorar las prácticas educativas.

\section{REFERENCIAS}

Aramburuzabala, P. (2013). Editorial monográfico aprendizaje-servicio. Revista Internacional de Educación para la Justicia Social, 2(2), 5-11.

Batlle, R. (2013). El aprendizaje-Servicio en España: el contagio de una revolución pedagógica necesaria. Madrid, España: PPC Editorial.

Bisquerra, R (2016). Metodología de la investigación educativa. Madrid, España: La Muralla.

Blanco, L. (2019, 15 de septiembre). Los alumnos de Formación Profesional crecen el doble que los de Bachillerato. [La Razón Web]. Accesible en: https://tinyurl.com/rm7sj34

Cadena Ser (2019, 15 de julio). Más de la mitad de los padres reconocen tener "mala imagen" de la F.P. [Notica Web]. Accesible en: https://tinyurl.com/yxxj5sg9

Deeley, S.J. (2016). El aprendizaje-servicio en educación superior: teoría, práctica y perspectiva crítica. Madrid, España: Narcea.

González, I. (2019, 28 de diciembre). La Formación Profesional sigue disparada y crece un 50\% desde 2010. [Diario de Navarra Web]. Accesible en: https://tinyurl.com/rxgtoxe

Heidegger, M. (2005). Ser y Tiempo (rustica). Granada, España: Editorial universitaria. 
Ley Orgánica 2/2006, de 3 de mayo, de Educación (LOE). Publicada en BOE nº 106 el 4 de mayo de 2006 (pp. 17158-17207).

Ley Orgánica 8/2013, de 9 de diciembre, para la Mejora de la Calidad Educativa (LOMCE). Publicada en BOE no 295 el 10 de diciembre de 2013 (pp. 97858-97921)

La Vanguardia (2019, 15 de julio). La FP sigue siendo el "patito feo" de la educación. [Noticia Web]. Accesible en: https://tinyurl.com/vbjynqv

Martínez, A. (2007). Service-learning o aprendizaje-servicio. La apertura de la escuela a la comunidad local como propuesta de educación para la ciudadanía. Bordón, 59 (4), 627-640.

Mayor, D., López, A.M. y Solís, M.G. (2019). El Aprendizaje-Servicio como Escenario Formativo y su Influencia en Distintos Agentes Socioeducativos. Percepción de los Participantes. International Journal of Sociology of Education, 8(2), 153-172. doi: 10.17583/rise.2019.4071

Mendia, R. (2016). El aprendizaje-servicio: una metodología para la innovación educativa. Revista Convives, (16), 1-16.

OCDE (2014). Education Policy Outlook Spain. Informe elaborado por la OCDE, accesible en: http://www.oecd.org/Spain/

Pascual, B. (2006). Calidad, equidad e indicadores en el sistema educativo español. Pulso, (29), 43-58.

Piedra, J.L. (2019, 25 de junio). La FP sigue lastrada por su mala imagen entre los padres en favor de la universidad. [Diario Sur Web]. Accesible en: https://tinyurl.com/qpjr67x

Real Decreto 1147/2011, de 29 de julio, por el que se establece la ordenación general de la formación profesional del sistema educativo. Publicado en BOE no 182 el 30 de julio de 2011 (pp. 8676686800).

Soler, M. (2014). Una mirada sobre la educación. La formación profesional. Revista padres y maestros, 359, 41-44.

Suárez, B. y Castillo, I. S. (2020). Descripción de una experiencia educativa inclusiva con alumnado universitario: Trabajando habilidades para el empleo. Tendencias Pedagógicas, 35, 130-152. doi: 10.15366/tp2020.35.11

Tapia, N. (2006). Aprendizaje y servicio solidario: algunos conceptos básicos. Documento incluido dentro de la Biblioteca Digital de la Iniciativa Interamericana de Capital Social, Ética y Desarrollo del Banco Interamericano de Desarrollo (BID). Accesible en: www.iadb.org/etica

Torres, A. y Vallejo, M. (2018). ¿Contribuye la universidad al desarrollo de aprendizajes de calidad? Estudio descriptivo con estudiantes de la Universidad de Murcia. Revista Electrónica e Interuniversitaria de Formación del Profesorado, 21 (1), 129-142. doi: 10.6018/reifop.21.1.29526

Uruñuela, P.M. (2018). La metodología del aprendizaje-servicio. Madrid, España: Narcea.

Wood, P. y Smith, J. (2017). Investigar en educación. Madrid, España: Narcea.

1 Información disponible en la web del Ministerio de Educación y Formación Profesional: http://www.todofp.es

${ }^{2}$ El Catálogo Nacional de Cualificación Profesional está accesible a través del siguiente enlace: http://incual.mecd.es/bdc 